\title{
The optimization of the opto-mechanical performance of the mirror segments for the E-ELT.
}

\author{
Jan Nijenhuis, Roger Hamelinck, TNO, Stieltjesweg 1, 2623 CK Delft, The Netherlands.
}

\begin{abstract}
ESO is preparing to build the E-ELT which will be the largest optical telescope in the world. Its primary mirror will be $\varnothing 39.3 \mathrm{~m}$ and will consist of nearly 1000 hexagonal segments of 1.2 meter width. Each segment is to be supported by the same structure although the segments vary in size and shape, hence this will cause variable surface deflection. Its support structure must guarantee a maximum surface form error of $30 \mathrm{~nm}$ rms independent of the segment orientation and environmental temperature. Measures, such as mass balancing and active surface form compensation are needed to compensate for these effects.

To meet the main optical and mechanical performance requirements, numerous FEM analysis runs have been made. Dedicated software was used to calculate mirror deformations under mechanical loads and to express these in Zernike modes. Especially this tool proved to be very powerful in proving that the optical surface form requirements could be met for all different sized mirror segments.
\end{abstract}

Keywords: Opto-mechanics, ELT, statically determined, M1 support structure, Zernike, surface form deformations, warping harness.

\section{INTRODUCTION}

The E-ELT is going to be the biggest ground based optical telescope in the world in the coming decades. It will have a hexagonal diameter of $39.3 \mathrm{~m}$. The primary mirror will consists of nearly 1000 hexagonal shaped segments (Figure 1) with a minimal width of $1.2 \mathrm{~m}$. Because of the hexagonal shape of the segments the mirror can be subdivided in 6 identical sections. Due to the parabolic mirror shape all segment of a section will be different in size and mass. The challenge has been to design one type of support structure suitable for all segments.

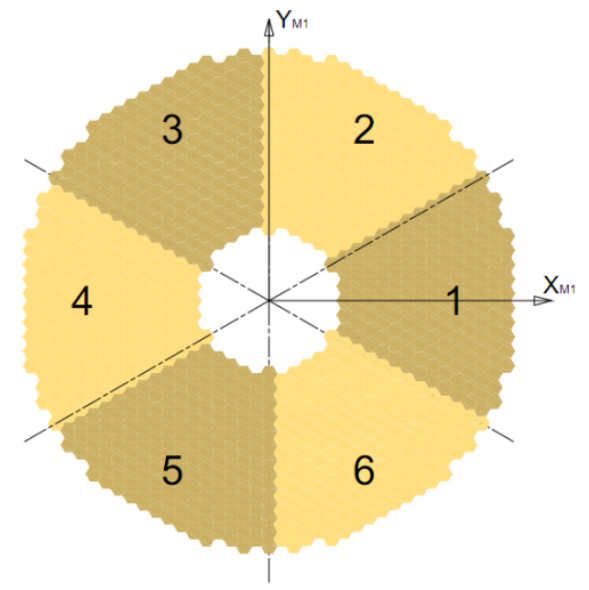

Figure 1: M1 segmentation.
To understand better the relation between the surface form analyses results and the support of the mirror segment first a brief description is given of this support. Figure 2 shows the classical whiffletree design. It is in 2D and it is the classical way to spread the load of the segment on its support points. Figure 3 shows the 3D version of the whiffletree structure. Each segment will be supported by three whiffletrees as shown in Figure 4. Such whiffletree consists of three tripods (2x Outer Tripod and 1x Inner Tripod) on top of another tripod named TLT (Top Level Tripod). This way the mass of the segment is spread over 27 support points. This reduces the surface form deformation of the mirror considerably. The optimal location of these points was determined and prescribed by ESO. The mechanical loading of the whiffletrees is determined by its geometry (static determined structure). However due to the out of plane bending stiffness of the segment the loading of the 27 support points becomes dependent again on the stiffness of the whiffletree. Furthermore parasitic bending moments caused by the elastic hinges of the whiffletree may also influence the surface form deformation.

Integrated Modeling of Complex Optomechanical Systems, edited by Torben Andersen, Anita Enmark, Proc. of SPIE Vol. 8336, 83360H - (C) 2011 SPIE · CCC code: 0277-786X/11/\$18 - doi: 10.1117/12.916685 


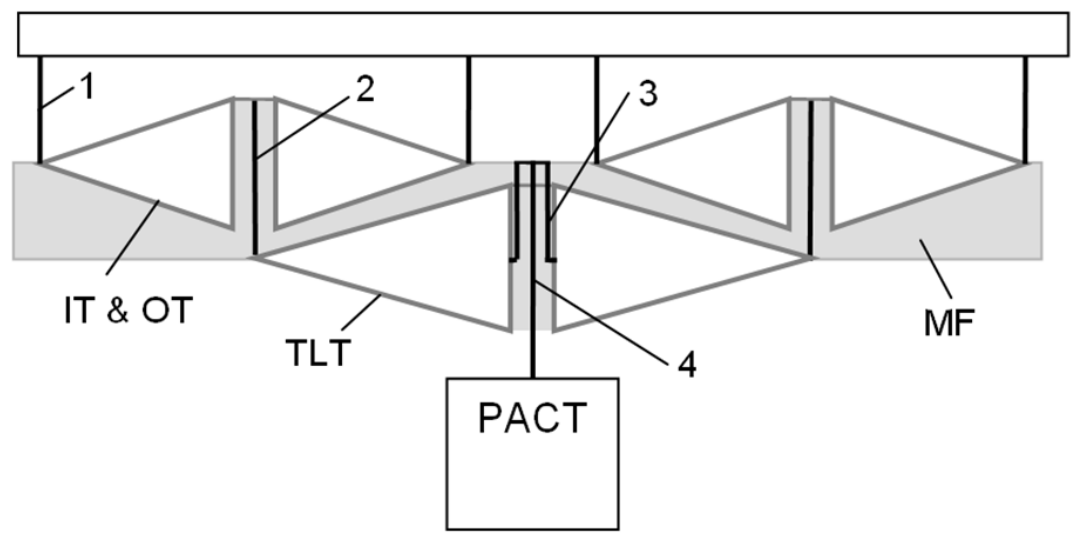

Figure 2: Classical whiffletree design (2D)

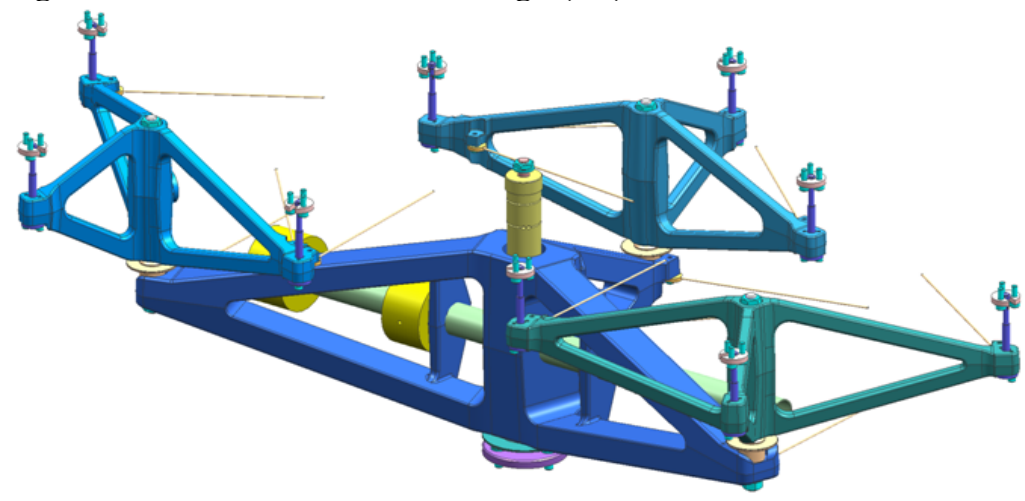

Figure 3.: 3D version of a whiffletree

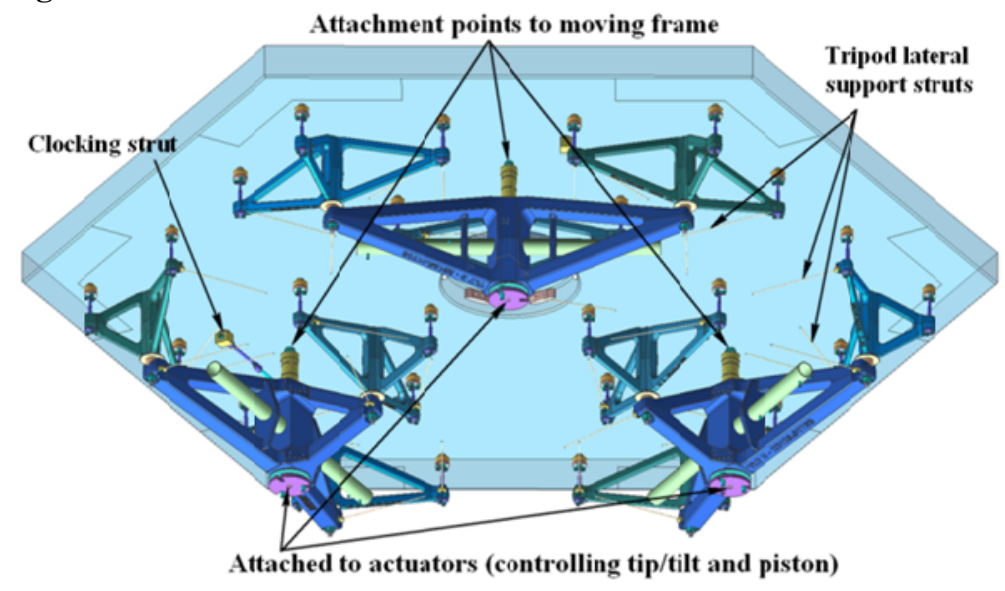

Figure 4: The whiffletree supports of the mirror segment.

The whiffletree only supports to the segment in the normal direction to the segment thereby constraining piston and tip/tilt. In addition a lateral support and clocking restraint is also needed to constrain all 6 Degrees Of Freedom (DOF) of the segment. A membrane is mounted in the center of the segment (Figure 5). The outer edge of this is bolted to the segment while the central part is bolted to the moving frame (Figure 6). This moving frame also provides the lateral support to the tripods. The loads of the whiffletrees are transferred to three actuators (PACT in Figure 2 and Figure 4) each supporting one of the whiffletrees. The moving frame as such forms an intermediate body that is connected to telescope structure. Its function is to isolate the segment from disturbing forces/moments (due to pointing variations of the telescope) that would otherwise deform the mirror segment. 


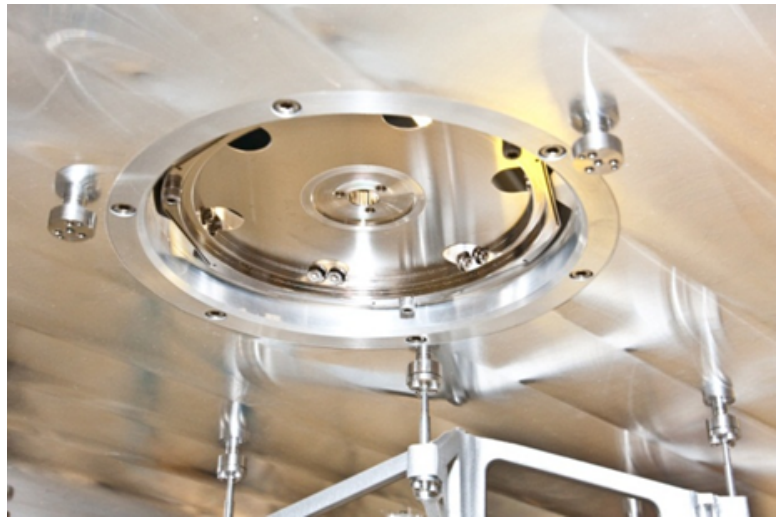

Figure 5: The membrane mounting into the dummy mirror segment.

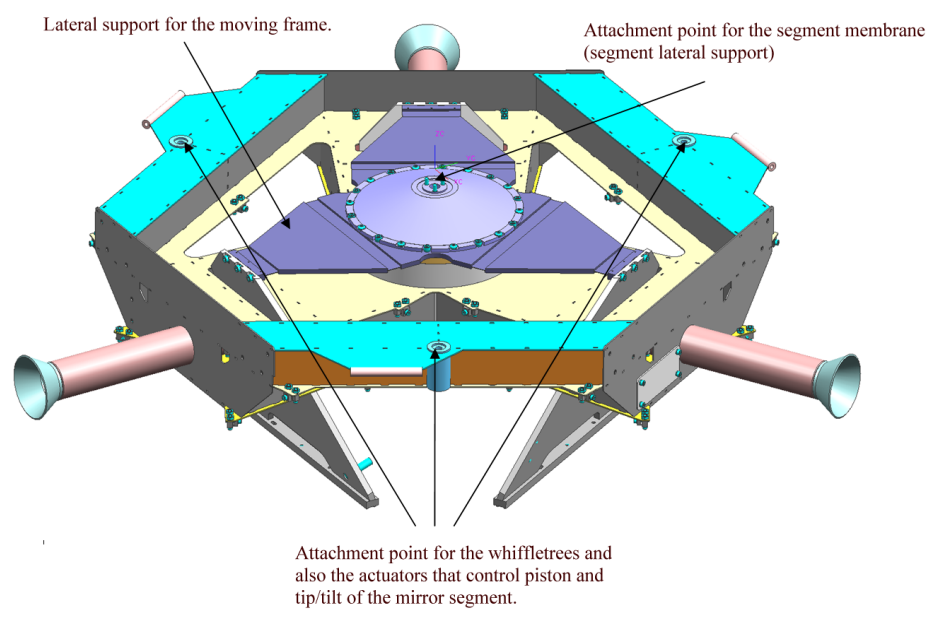

Figure 6: The moving frame holding the whiffletrees.

The main segment surface deformation requirements are:

1. The gravity imposed deformations should remain below $30 \mathrm{~nm} \mathrm{rms} \mathrm{(excl.} \mathrm{piston} \mathrm{and} \mathrm{tip/tilt)} \mathrm{taking} \mathrm{into} \mathrm{account}$ temperature changes of up to $30^{\circ} \mathrm{C}$.

2. This surface form deformation applies to tip/tilt angles of the telescope of $70^{\circ}$ max.

3. These requirements apply to all different segment sizes although passive compensation for segment deformation is allowed.

\section{ANALYSIS OF THE SEGMENT SURFACE FORM DEFORMATION}

By making an FE-model of the segment and its support structure (Figure 7) the surface form deformation of the mirror segment can be calculated as function of the tilt angle of the telescope. A typical result of this analysis is shown in Figure 8 . The deformed segment obviously shows a lot of tilt ( $8 \mu \mathrm{m}$ for a segment width of $1.2 \mathrm{~m})$. However it is not clear whether the surface of the mirror segment also shows other types of deformation because this is obscured by the large amount of tilt. Tilt will be compensated for by the three Position ACTuators (PACT).

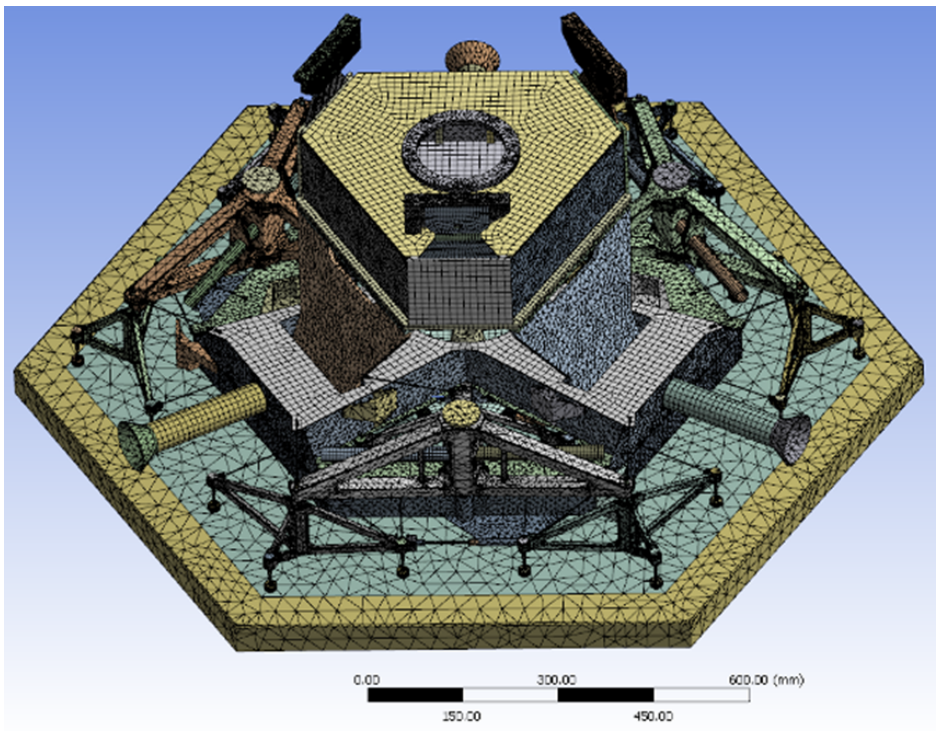

Figure 7: The FE-model of the segment support structure.

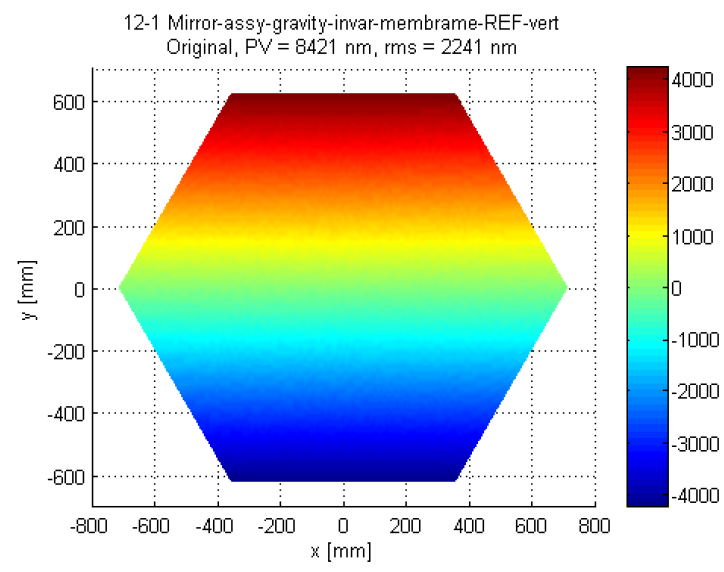

Figure 8: The deformation of the mirror segment surface due to gravity (mirror in vertical plane). 
A similar situation exists for the design of the lateral support of the mirror segment (Figure 5). Metal and glass are connected to each other and the thermal consequences of that are counteracted by using flexures. Does that mean that any type of metal can be used? Invar matches best which the CTE of the segment but is expansive the lateral support becomes difficult to manufacture. Stainless steel is cheap but it may cause a problem due to the difference in thermal expansion with the glass mirror segment. Analysing this for these two metals gives the results as shown in Figure 9 and Figure 10.

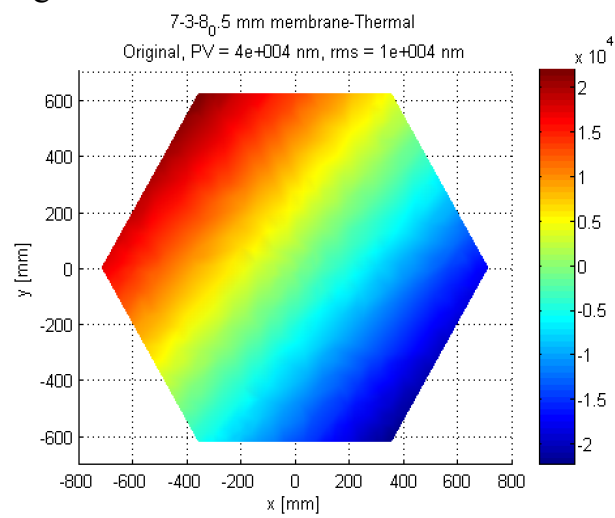

Figure 9: Mirror surface form deformation after temperature increase using stainless steel membrane (no gravity load included).

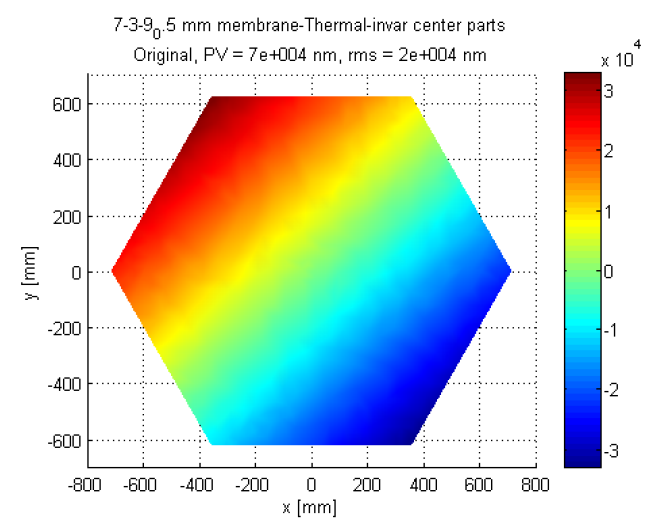

Figure 10: Mirror surface form deformation after temperature increase using invar membrane (no gravity load included).

It can be concluded that there is a difference in resulting tilt of the mirror when the environmental temperature changes. However this can be compensated for by the three supporting actuators. Further conclusions are difficult to make.

\section{SURFACE FORM ANALYSIS TOOL DESCRIPTION.}

Opticians often use Zernike terms to mathematically describe the surface form of an optical component. E.g. it is used to trace where improvements in the optical design are possible. The Zernike mode shapes form an orthogonal system (that means that each surface form can only be described in one way with Zernike modes). The lower order Zernike mode shapes are shown in Figure 11 with their names. Only these are important for mechanical purposes. The number of possible mode shapes is infinite. The results of the Zernike de-composition can be used by the mechanical engineer to link a specific surface form deformation to specific structural elements.
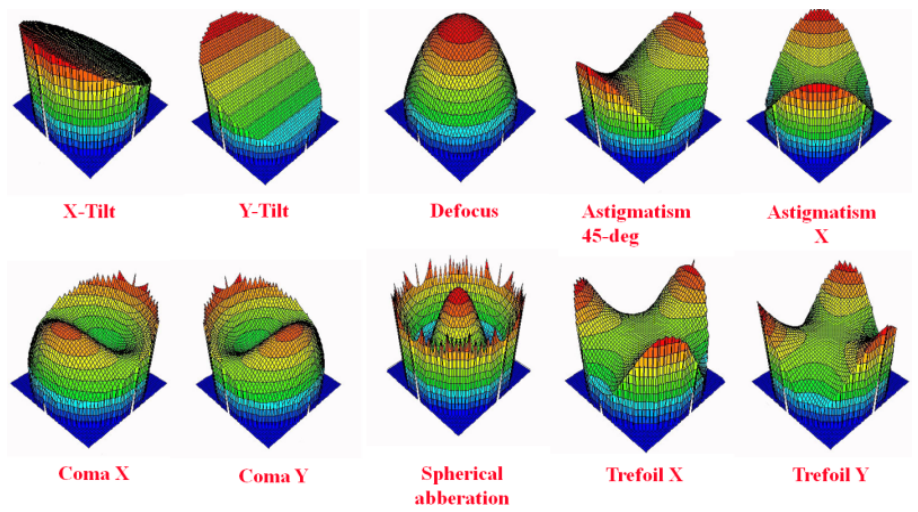

Figure 11: Surface form de-composition into its Zernike modes.

Zernike de-composition of surface forms has been used for decades by opticians. The Zernike de-composition tool is generally implemented in optical analysis software and therefore not easily assessable for mechanical engineers. TNO has build a surface form de-composition tool using Matlab. With this tool the mechanical engineer is able to analyse the surface form deformation as shown in e.g. Figure 8. Results are shown in Figure 12. It shows the large contribution of 
piston, tip/tilt as well as astigmatism and coma. The rest is a small residual error. Figure 13 shows the original surface form (i.e. Figure 8), the surface form after piston and tip/tilt subtraction and finally also without focus. It is presented in plane-view and 3D view to facilitate the user. The result after piston and tip/tilt subtraction is striking. Only $7.4 \mathrm{~nm} \mathrm{rms}$ remains meaning that the surface form is nearly perfect and well within the ESO specification of $30 \mathrm{~nm} \mathrm{rms}$.

At the centre of the segment some local tilt is visible causing the astigmatism and coma. Although the membrane coincides with the COG of the segment the moving frame will tilt slightly and will induce a small local tilt of the membrane. The membrane has only $0.5 \mathrm{~mm}$ thickness and its bending stiffness is very low. Still the effect is not zero as becomes clear from Figure 13. This clearly illustrates the strength of the tool.
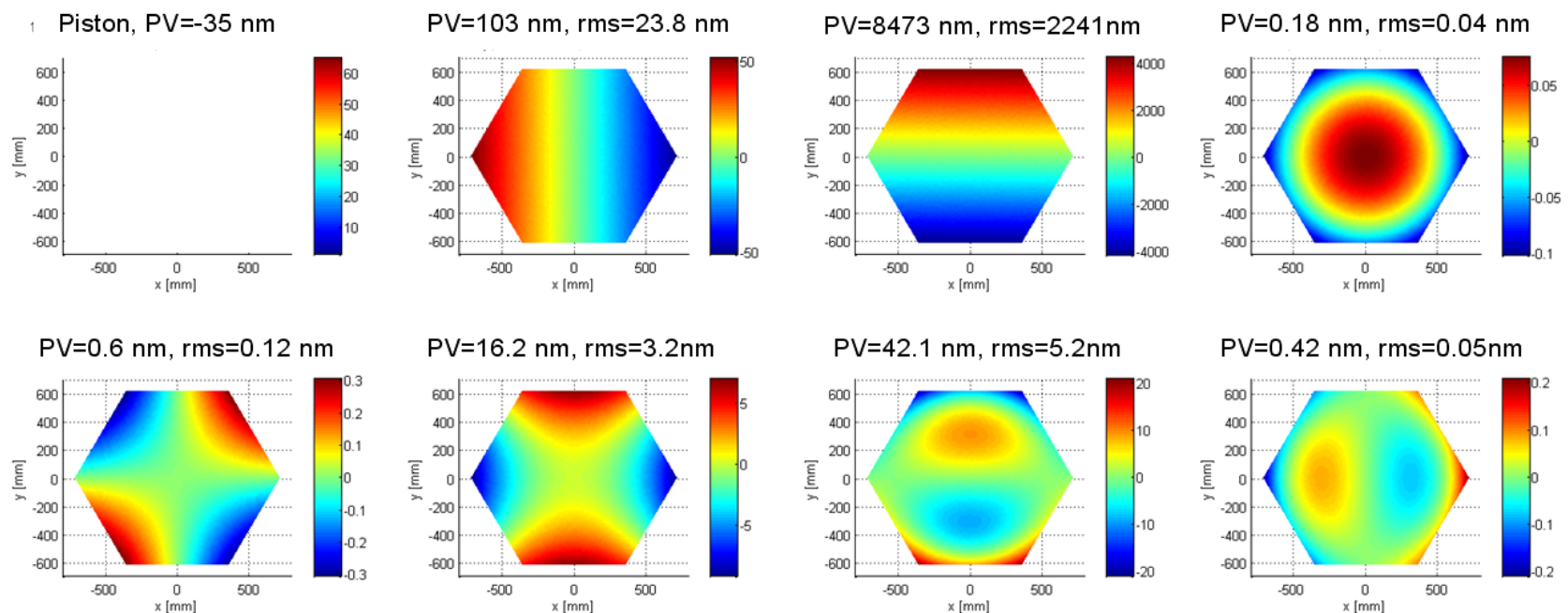

$P V=0.42 \mathrm{~nm}, \mathrm{rms}=0.05 \mathrm{~nm}$

$\mathrm{PV}=0.25 \mathrm{~nm}, \mathrm{rms}=0.05 \mathrm{~nm}$
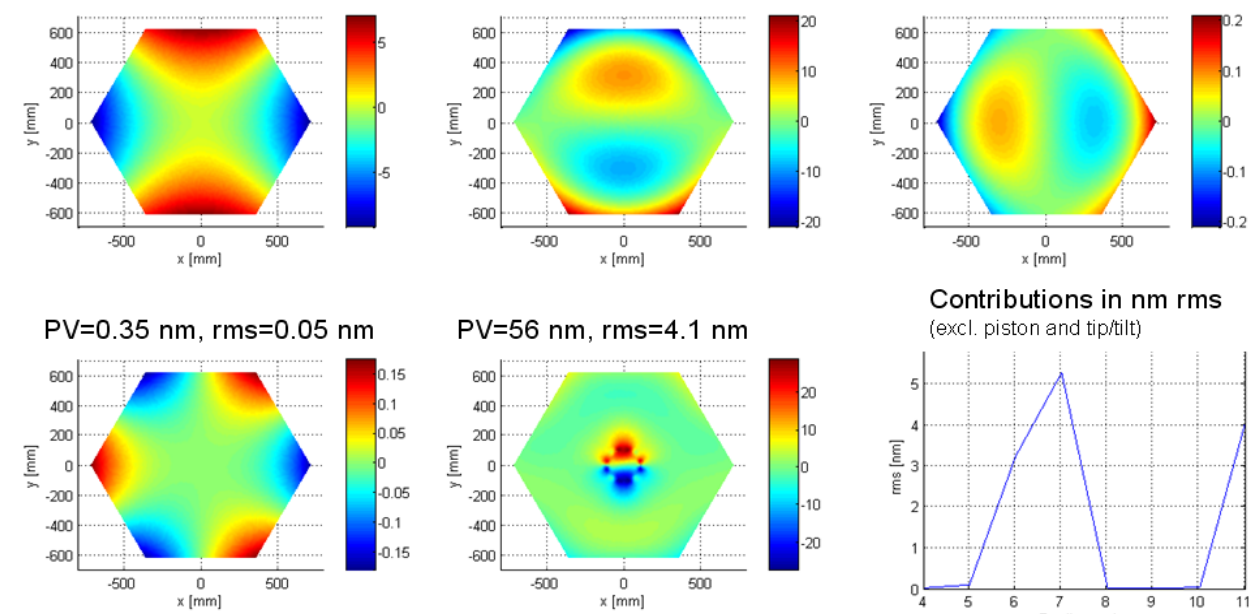

Contributions in $\mathrm{nm}$ rms (excl. piston and tip/tilt)
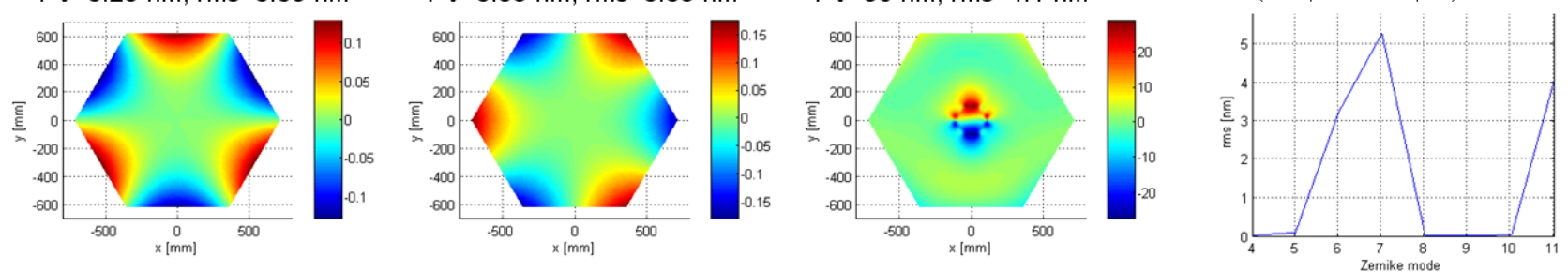

Figure 12: Zernike decomposition of the surface acc. Figure 8. 

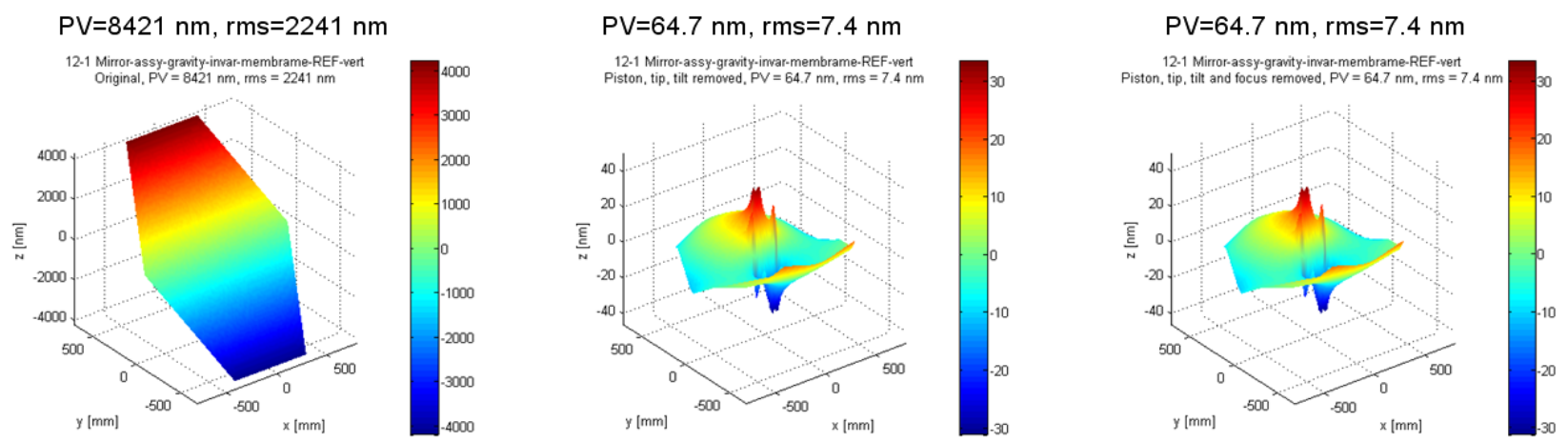

$\mathrm{PV}=8421 \mathrm{~nm}, \mathrm{rms}=2241 \mathrm{~nm}$
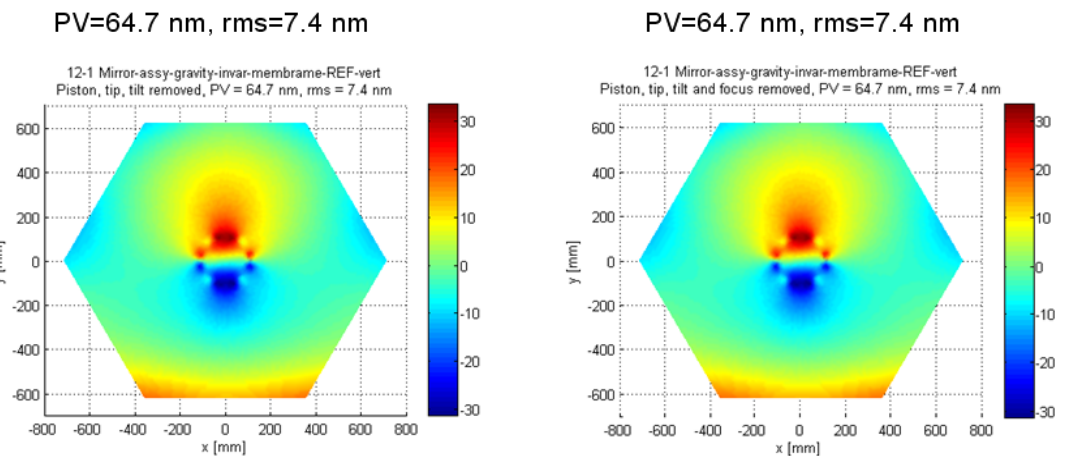

Figure 13: Surface form deformation of the segment with various Zernike mode shapes subtracted.

Similarly the results from Figure 10 and Figure 11 have been analysed resulting in Figure 14 and Figure 15. Now it becomes evident that a stainless steel membrane is unacceptable because a temperature change of only $10^{\circ} \mathrm{C}$ already causes a surface form error, which is close to the total budget. Please note that the membrane is connected to the mirror segment using six leaf springs (located at equal distances along the circumference of the membrane) that are intended to isolate the difference in thermal expansion from each other. It is remarkable to see that the bending stiffness of these leaf springs still cause a considerable amount of surface form deformation.

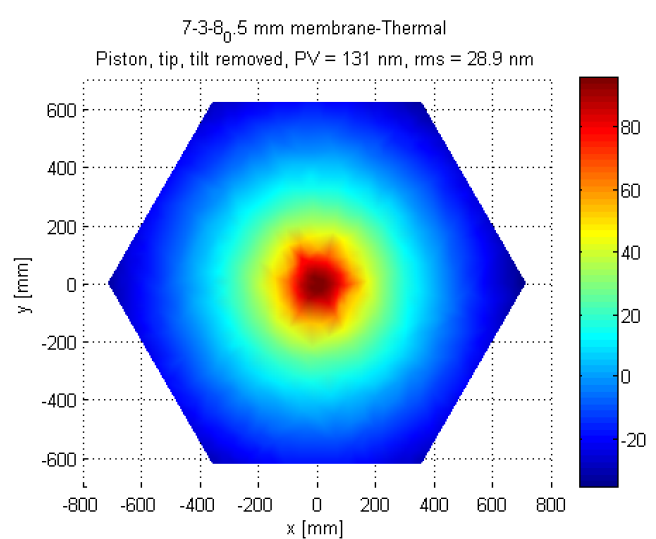

Figure 14: As Figure 9 after removal of piston and tip/tilt.

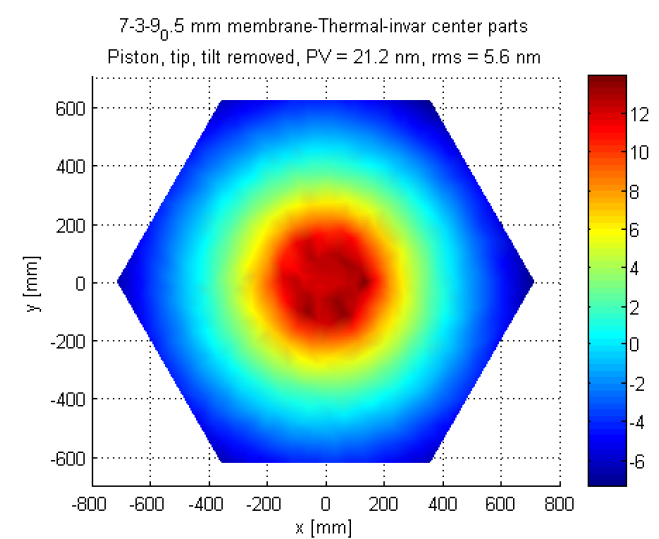

Figure 15: As Figure 10 after removal of piston and tip/tilt.

Another example of the strength of the Zernike tool is illustrated in Figure 16 (mirror segment not shown for clarity). One of the actuators is displaced by $2 \mathrm{~mm}$. The consequence will be that mirror segment, whiffletrees and moving frame together will move as one solid body. This motion will be a translation in combination with some tilt when referencing 
this to the segment centre. This tilt action will cause that the lateral support of the moving frame (Figure 6) will deform (see Figure 17) by bending (LH-side) and torsion (other two leaf springs). These deformations will caused reaction loads in the moving frame and question is whether this is visible in the mirror segment surface.

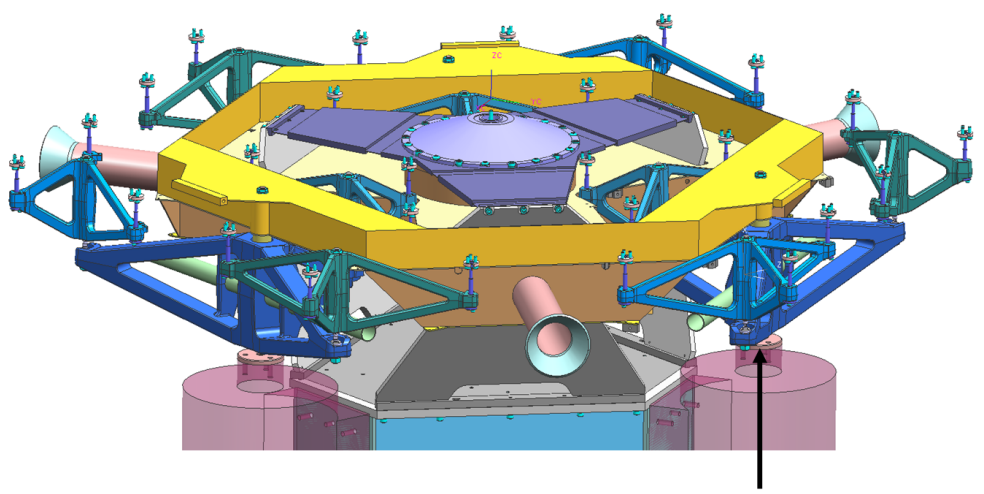

Applied displacement

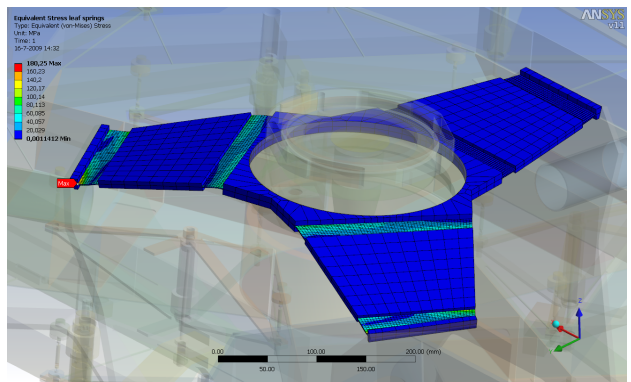

Figure 17: Calculated deformations of the lateral support of the moving frame.

Figure 16: Surface form deformation resulting from single actuator stroke (2mm).

Original deformed surface

$P V=1368 \mathrm{~nm}, \mathrm{rms}=292 \mathrm{~nm}$

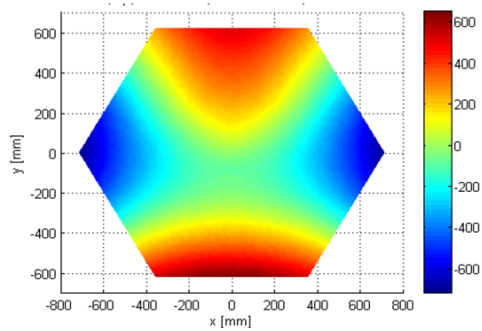

Figure 18: Surface deformation resulting from actuator displacement.

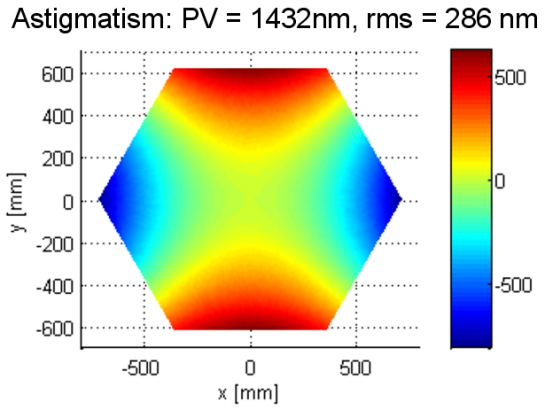

Figure 19: Main contributor in surface form deformation.

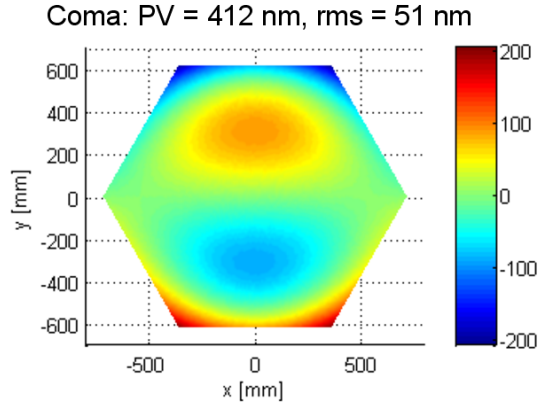

Figure 20: Second contributor to surface deformation.

The answer is given in Figure 18. $292 \mathrm{~nm}$ rms was found as surface form deformation. From Figure 19 and Figure 20 it can be concluded that astigmatism and coma are the main contributors and these can be directly linked to the leaf spring deformations. Therefore the bending and torsion stiffness of these leaf springs need to be reduced without changing the shear stiffness (which is directly linked to the stiffness of the lateral support of the moving frame). Changing the leaf spring parameters can do that. This way the astigmatism and coma completely disappeared.

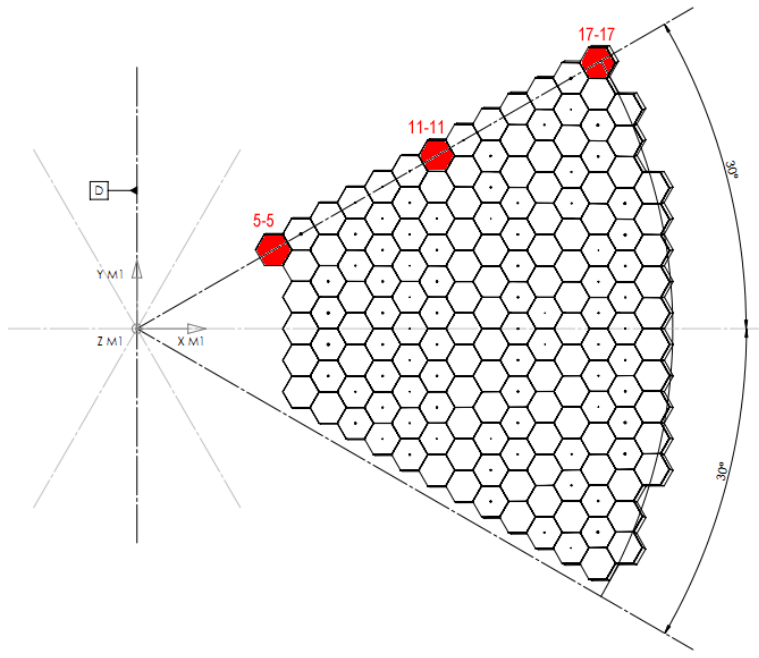

\section{COMPENSATION OF SURFACE FORM DEFORMATIONS.}

Only one type of support structure is to be used for all types of mirror segments. That will cause differences in surface form deformation. Three segments were analysed as shown in Figure 21. The most outer segment is the biggest. Figure 22 Figure 24 show the differences in deformation after subtraction of piston and tip/tilt. From the results it can be concluded that the differences in deformation are unacceptable. Hence some way of compensation is needed. 
In Figure 2524 options are presented to apply moments to the whiffletrees to compensate for surface form deformations. The moments are always applied in the radial or tangential direction. Figure 26 gives an impression how this is done. A self-locking motor drives an output shaft that (un) winds a clocking spring to realise the required moment.

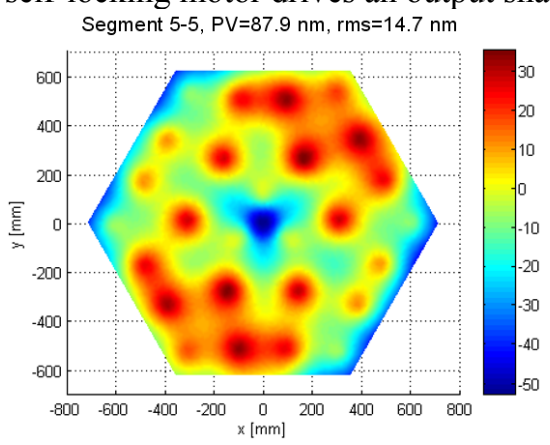

Figure 22: Inner segment deformation.

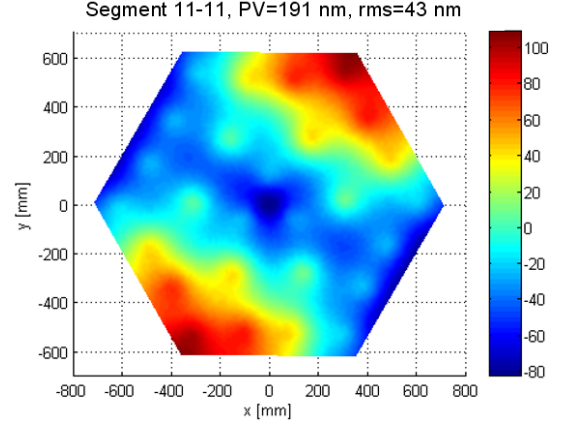

Figure 23: Middle segment deformation.

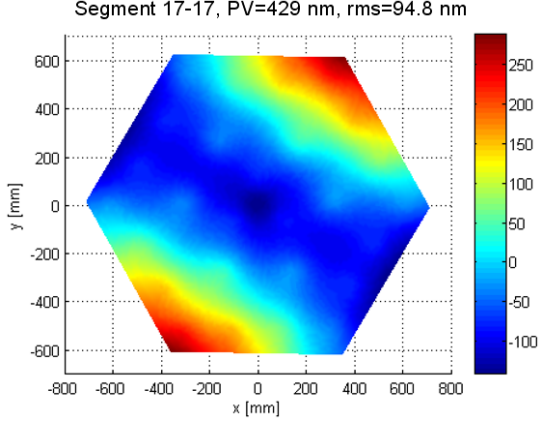

Figure 24: Outer segment deformation.

The actuator is attached to the moving frame while the other end is connected to the tripod.

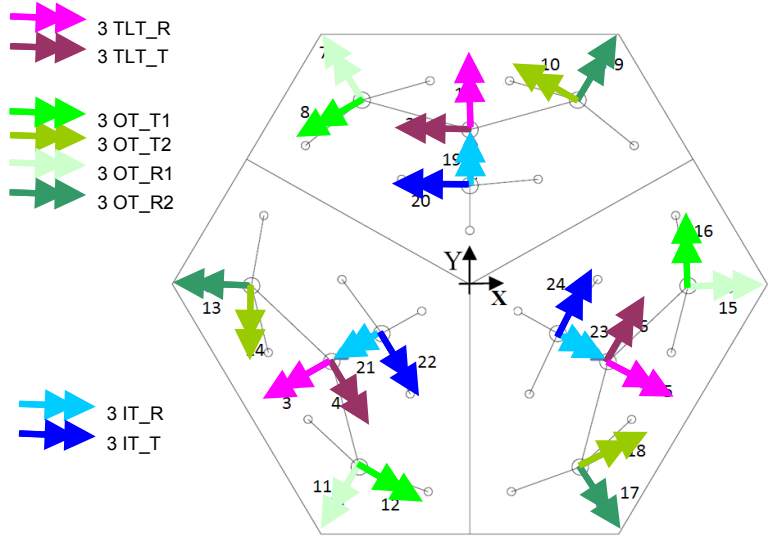

Figure 25: 24 options to apply correction moments to the segment.

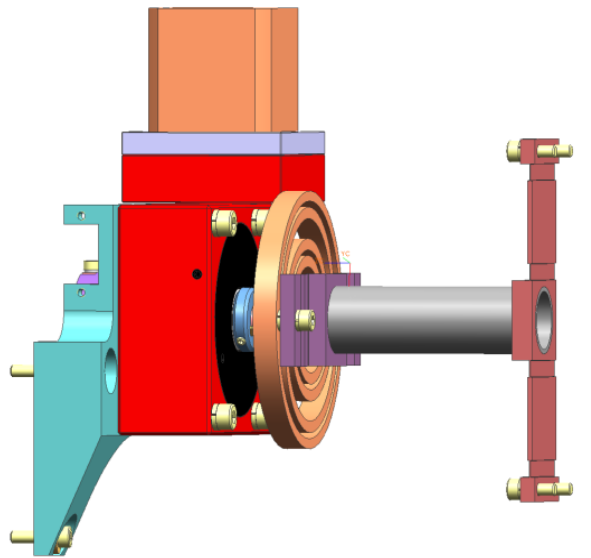

Figure 26: Self locking motor to apply correction moment.

A unit moment is applied at each of the 24 positions and the corresponding surface form deformations are calculated using the FE-model of Figure 7. This provides the influence functions as shown in Figure 27. By combining these influence functions each individual Zernike mode can be made.
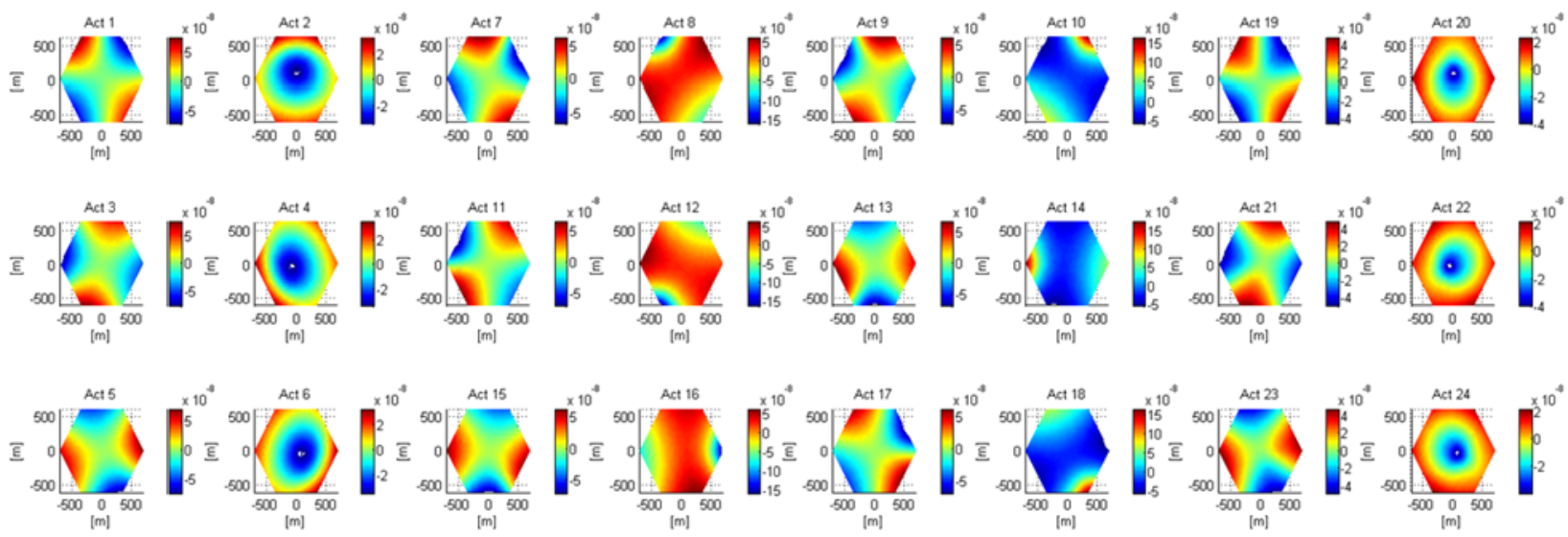

Figure 27: Influence functions of all 24 correction options. 
How well this can be done is shown in Figure 28. The first row shows the various Zernike mode shapes (focus, astigmatism $45^{\circ}$ and astigmatism $\mathrm{X}$, coma $\mathrm{X}$ and coma $\mathrm{Y}$, trefoil $\mathrm{X}$ and trefoil $\mathrm{Y}$ ). The second row shows how well they can be approximated with a linear combination of the 24 influence functions. When the two are subtracted the final result is shown in the third row. E.g. for focus a reduction with a factor 18.5 is realized i.e. the ratio of the rms wavefront error prior and after correction. For the others the improvements are factors 59.5, 57.6, 5.2, 5.5, 7.8 and 36.6. From this it can be concluded that focus, astigmatism and trefoil Y can be well compensated.

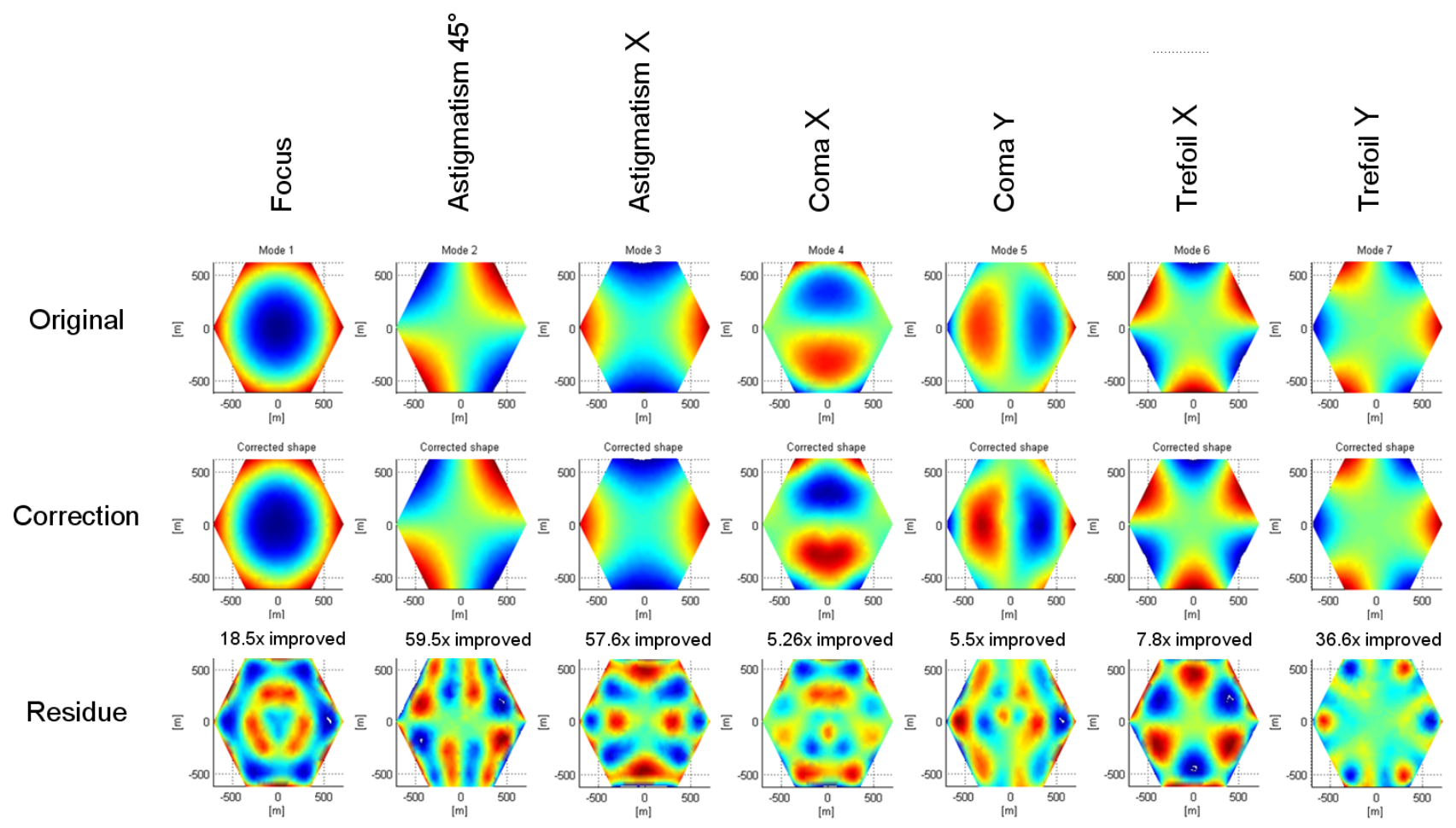

Figure 28: Resulting surface form deformation after correction for specific Zernike mode.

From a practical point of view it is desirable to use the least amount of torsion actuators. Not all locations are equally efficient. That is why a study has been made to determine the minimum number of actuators that can provide improvement factors of 7, 18 and 6 for focus, astigmatism and trefoil. Ten actuator combinations have been analysed arriving at the conclusion that the configuration as shown in Figure 29 is best. 


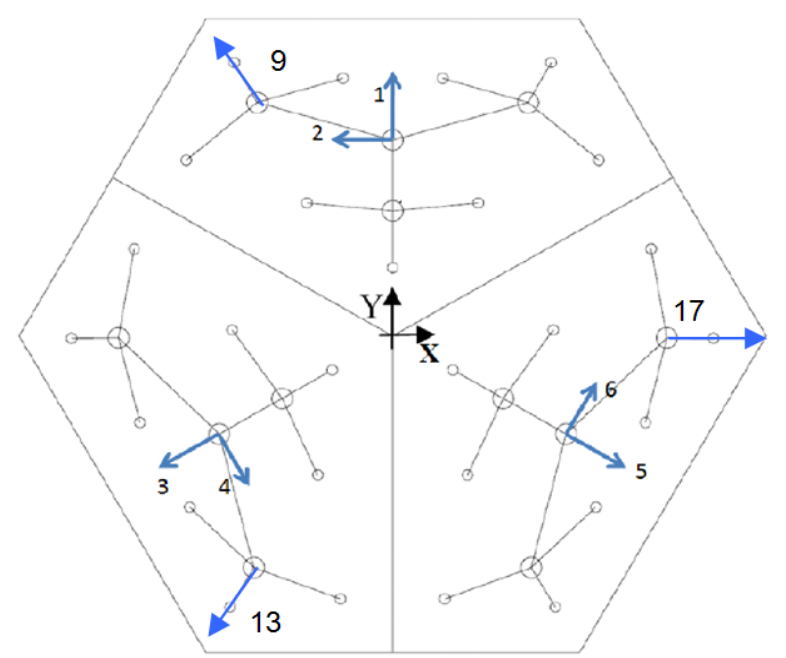

Figure 29: The required locations where moments are applied to meet the surface form requirement.

The results from Figure 22 - Figure 24 improve to what is shown in Figure 30 - Figure 32when using the influence functions of the torsion actuators as shown in Figure 29. Only a small difference between the mirror segment results exists and the remaining surface form error remains well within the requirement. It can therefore be concluded that it is well possible to support all mirror segments with the same support structure using only nine correcting moments at locations as shown in Figure 29.

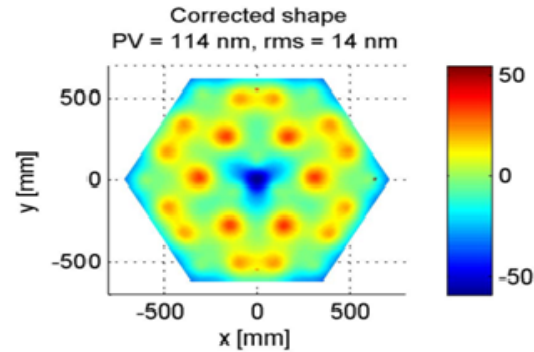

Figure 30: Inner segment deformation after surface form correction.

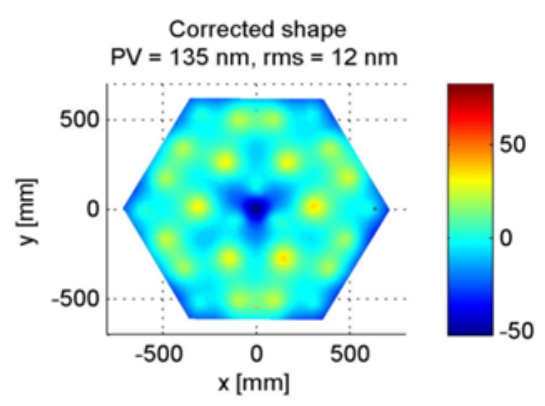

Figure 31: Middle segment deformation after surface form correction.

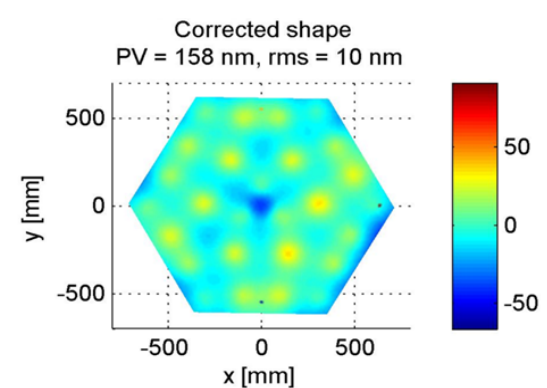

Figure 32: Outer segment deformation after surface form correction.

\section{CONCLUSIONS}

Conclusions that can be drawn from the performed surface form analysis for the mirror segments of the E-ELT are:

- FEM-analysis is a general tool that needs specific additional software to reveal the full content of the calculated deformations. This is illustrated by few examples in this article.

- The decomposition of the surface deformation into Zernike mode shapes has proven to be a powerful design tool to be used in design trade-offs.

- Zernike mode decomposition allows tracing where specific surface form deformations are orienting from.

- The unit sensitivity matrices allowed calculating the required surface form corrections.

- It can be concluded that all mirror segments can be supported by the same support structure provided static or dynamic balancing is done using nine actuators at the locations as indicated in Figure 29. 